\title{
Role of 2-dimensional speckle tracking echocardiography in diagnosis of coronary artery stenosis instable angina pectoris patients
}

Background and methods: In this work, 120 patients suspected of having stable angina pectoris were included. They were presented for evaluation of chest pain and to whom clinical evaluation, echocardiography, nuclear scanning and coronary angiography were done. They were classified into group (A) 40 control patients considered as a control group with normal coronaries, and group (B) 80 patients with significant CAD.

Results: The study showed that regarding the Echo. Parameters, there were statistically significant difference between the 2 groups regarding the A wave, E/A ration, DT, Em and E/EM. Also regarding SLSS and GLS 17 and GLS 12 as well as SLSr, GLSr 17 and GLSr 12. Significant difference was present regarding number of vessels affected in regard to GLS 12, GLSr 12 and GLSr 17. In comparison with the results of MPI, there was a positive correlation between the number of segments affected in MPI and GLS 12 and GLSr 12. A statistically significant correlation was also found between the 17 segments in MPI and SLSS and SLSr parameters.

Conclusion: Myocardial strain by speckle tracking is superior to conventional Echo. Parameters measurements of global and segmental LS using 2DSE and it is a more sensitive tool in the identification of WMA at rest than visual analysis and that support its use to risk stratify atherosclerotic CAD. It may help in identifying which coronary artery is affected.

Keywords: Speckle tracking; Myocardial perfusion scan; Coronary artery disease; Comparative study

Echocardiography is the leading cardiac imaging technique in patients with suspected cardiac disease. However, conventional echocardiography at rest provides little information regarding the presence and extent of coronary artery disease (CAD) in patients suspected of suffering from stable angina pectoris (SAP) [1]. Longitudinally orientated myocardial fibers are located subendocardially, the area most susceptible to ischemia, that is why measurements of longitudinal motion and deformation may be the most sensitive markers of CAD using tissue Doppler imaging (TDI) or two-dimensional strain echocardiography (2DSE) [2]. The analysis of myocardial deformation has emerged as a quantitative means of reliably estimating myocardial contractility. Stress imaging techniques have several advantages over conventional exercise ECG testing including superior diagnostic performance for the detection of obstructive coronary disease, the ability to quantify and localize areas of ischemia, and the ability to provide diagnostic information in the presence of resting ECG abnormalities or inability of the patient to exercise [3]. So the aim of this study was to compare and determine the correlation between 2DSE at rest and myocardial perfusion imaging (MPI) examination in diagnosis of CAD instable angina pectoris patients.
Ehab E El-Hefny*, Ibraheem and Moustafa Eldeib

Department of Cardiology, Al Azhar University, Egypt ${ }^{*}$ Author for correspondence: Tel: 00201066008846 ehabelhefny@hotmail.com Submitted: 14 November 2016 Accepted: 06 December 2016 Published online: 12 December 2016 


\section{Patients and Methods}

\section{Study population}

The present study included (120) patients suspected to be stable angina pectoris patients. All patients were presented for evaluation of chest pain at our outpatient clinics, Echocardiography, cath lab and nuclear units at Al-Hussein and Bab EL-She'riya University HospitalsAl-Azhar University-Cairo-Egypt, between December 2013 and December 2015, were considered to participate in this study.

The patients were classified according to coronary angiography results into two groups: Group (A) (control group): 40 patients with normal coronary angiography considered as a control. Group (B) (patient group): 80 patients with significant coronary artery disease, their ages range from 40 to 58 years. Inclusion criteria: Stable angina pectoris was defined as chest pain or discomfort (angina) suspected to be due to myocardial ischemia. Symptoms of angina will be considered stable if they have been occurring over several weeks without deterioration and typically induced by activity or stress. Exclusion criteria: Acute coronary syndrome patients, prior myocardial infarction, prior coronary interventions, left ventricular ejection fraction $\leq 50 \%$, significant regional wall motion abnormalities, congestive heart failure, atrial fibrillation, significant valvular heart disease, cardiomyopathies, congenital heart disease, technically poor acoustic window for transthoracic echocardiography, Patients with contraindication for exercise myocardial perfusion exam. Informed consent was taken from all patients. Complete history and full physical examination were done to all patients. The history highlighted the duration of chest pain, diabetes mellitus, smoking, hypertension, drug history and family history.

Full clinical examination was carried out on every patient. Resting standard 12-leads electrocardiogram searching for rate, rhythm, BBB and chamber enlargement and ischaemic changes,

Echocardiography: A Philips IE 33 X Matrix phased array system equipped with TDI \& STE technology, using a multi frequency $(1-5 \mathrm{MHz}) \mathrm{S} 5-1$ matrix array probe was used. All patients were examined from the standard views (parasternal long-axis, parasternal short axis at papillary muscle level, apical four -chamber, apical five -chamber and apical two-chamber). Recordings and calculations of different cardiac chambers and ejection fractions were made according to the recommendations of the American Society of Echocardiography [4]. Left ventricular (LV) systolic function: by calculating Ejection Fraction (EF) using M-mode cube method.

From apical four-chamber view pulse wave Doppler Mitral inflow velocities was recorded by placing sample volume at the tips of the Mitral valve leaflets, recordings obtained from the mitral annulus, between the body of the leaflets orient the image such that the transducer beam is parallel to flow (color flow Doppler may be used to optimize beam placement) sweep speed $50-100 \mathrm{~mm} / \mathrm{s}$, for measurement of peak early diastolic velocity (E) wave, peak late diastolic velocity (A) wave, $\mathrm{E}$ wave deceleration time (DT), E/A ratio were calculated

Pulsed tissue Doppler parameters: The mitral annular velocities were recorded using the pulsed-wave TDI. A variable frequency phased array transducer $(1-5 \mathrm{MHz})$ was used. The filter settings were kept low $(50 \mathrm{~Hz})$ and gains were adjusted at the optimal level for good quality velocity. A $1.7 \mathrm{~mm}$ sample volume was used, adjusting the spectral pulsed Doppler signal filters until a Nyquist limit of $15-20 \mathrm{~cm} / \mathrm{s}$, and using the minimal optimal gain. A mean of three consecutive cycles was used to calculate all Echo-Doppler parameters, the sample volume is typically placed in the ventricular myocardium immediately adjacent to the mitral annulus to assess the diastolic function, the peak early diastolic velocity (Em) and peak late diastolic velocity $(\mathrm{Am})$ at Mitral annulus was determined. For lateral and septal sites apical 4chamber view was utilized, Mean values from above two sites were used to assess global diastolic left ventricular function [5], By Tissue Doppler we measured: Early diastolic annulus velocity (Em), Mean value of Em: Mean values from above two sites were used to assess global diastolic left ventricular function, E/Em: was measured using E wave of mitral inflow by pulsed wave Doppler over mean of Em at septal and lateral mitral annulus by pulsed tissue Doppler [6].

Speckle tracking Echocardiography study: all studies were done before coronary angiography. The following views were taken for later analysis; apical 4 and apical 2 chambers views and apical long axis view. In blinded post-processing, longitudinal deformation had been assessed by speckle tracking by measuring segmental peak systolic longitudinal strain (SLSS) and strain rate (Sr) for the 17 segment LV model from the apical 4chambers, 2-chambers and long axis views, with high frame rates (> 60 frames/s) using commercial imaging analysis software (Philips IE 33 software). End-systole was defined as aortic valve closure in the apical longaxis view by continuous Doppler wave recording. Automated delineation of endocardial borders was obtained through marking the mitral annulus level and at the apex on each digital loop. The area of interest was manually adjusted if automated delineation was not optimal. Segments with poor image acquisition or artifacts were excluded due to inability to measure LS segmental longitudinal peak systolic strain (SLS) closing for the basal, mid ventricular and apical segments and averaged from the all segments to provide global peak longitudinal systolic strain (GLS) for the 17 and 12 segments. The average from all 
segments to provide $\mathrm{Sr} 17$ and 12 segments were measured [7].

Myocardial perfusion scan: Single-photon emission computed tomography Gated-SPECT studies were performed using a two-day Stress/rest protocol for detection of myocardial ischemia. Patients underwent qualitative and semi quantitative SPECT, following intravenous administration of 20-25 mCi Tc-99m Sestamibi at peak exercise. In the next day, intravenous administration of 20-25 mCi under resting condition. Computed tomography images were acquired one hour after infusion of the radiotracer. Image acquisition was achieved with a dual head gamma camera (Siemens) without attenuation or scatter correction, using a low energy all-purpose collimator. Transaxial tomograms were reconstructed for each patient, at short-axis, horizontal and vertical long axis slices and were analyzed. A total of 17 myocardial segments per patient were studied and all images were reviewed by two experts; both were unaware of the other reading results by qualitative and semiqualitative analysis. Topographic images were collected from three planes: Short axis slices from apex to base of the heart were divided into four apical segments (apical anterior, apical septal, apical inferior and apical lateral), six mid-ventricular segments (mid anterior, mid antero-septal, mid infero-septal, mid inferior, mid posterior and mid lateral), and six basal segments (basal anterior, basal antero-septal, basal infero-septal, basal inferior, basal posterior and basal lateral). Vertical long axis slices from septum to lateral wall. The apical portion of the slice midway between the septum and lateral wall represents the apical segment resulting in a total of 17 segments for every patient (16 from short axis and 1 from the vertical long axis views) without duplication of the same anatomic area on different projection, for assessment of myocardial ischemia [8].

Coronary angiography: Coronary angiograms were obtained for each coronary vessel in at least two projections. Lesion locations were assessed and percent diameter stenosis was measured for each coronary lesion according to the American Heart Association classification. We assessed the number of affected vessels, using a cutoff of percent diameter stenosis $\geq$ $70 \%$ for the three epicardial vessels and $\geq 50 \%$ for LM coronary artery. The analysis of the coronary angiograms was performed visually by an experienced operator who was blinded to the results of the echocardiographic examinations [9].

\section{Statistical analysis}

The collected data were revised, organized, tabulated and statistically analyzed using statistical package for social sciences (SPSS) version 23.0 for windows. Data are presented as the Mean \pm standard deviation (SD), frequency, and percentage. Categorical variables were compared using the chi-square $(\chi 2)$ and Fisher's exact tests (if required). Continuous variables were compared by the Student $\mathrm{t}$ test (two-tailed) and one - way ANOVA test for parametric data with Bonferroni post hoc test to detect differences between subgroups, Mann-Whitney $U$ and Kruskal-Wallis tests for nonparametric data. Kendall's tau-b and Pearson correlations were used to study the correlation between the studied variables. Univariate logistic regression analysis was used to predict the presence of CAD among the studied sample. ROC curves were constructed to determine the cut - off values for diagnosing CAD among the studied sample. The level of significance was accepted if the $\mathrm{P}$ value $<0.05$.

\section{Results}

In this study there was no statistically significant difference between the two groups as regard of age, sex, heart rate, hypertension, diabetes mellitus, smoking and character of chest pain, as well as LVEDD, LVESD, LV EF\% and LVFS\%. There was statistically significant difference between the two groups in relation of $\mathrm{E}$ wave, $\mathrm{A}$ wave, $\mathrm{E} / \mathrm{A}$ ration, DT, $\mathrm{Em}$ and E/Em. There was no statistically significant difference between patients with single vessel disease, $v s$ two vessel disease, and multi vessel disease as regard of the Echocardiographic and tissue Doppler data (Table 1).

\begin{tabular}{|l|l|l|}
\hline \multicolumn{3}{|l|}{ Table 1: Total number of affected segments in MPI. } \\
\hline Segments & $r$ & $P$ \\
\hline GLS17 & 0.05 & 0.657 \\
\hline GLS12 & 0.295 & 0.008 \\
\hline Sr17 & 0.163 & 0.147 \\
\hline Sr12 & 0.286 & 0.01 \\
\hline
\end{tabular}

In this study, there was statistically significant difference between the two groups regarding SLSS and GLS 17 and GLS 12, as well as SLSr and GLSr 17 and GLSr 12. In the meanwhile, there was statistically significant difference between patients with single vessel disease vs two vessel disease and multi vessel disease in regard of GLS12 but not regarding GLS17 and SLSS (Figure 1). 


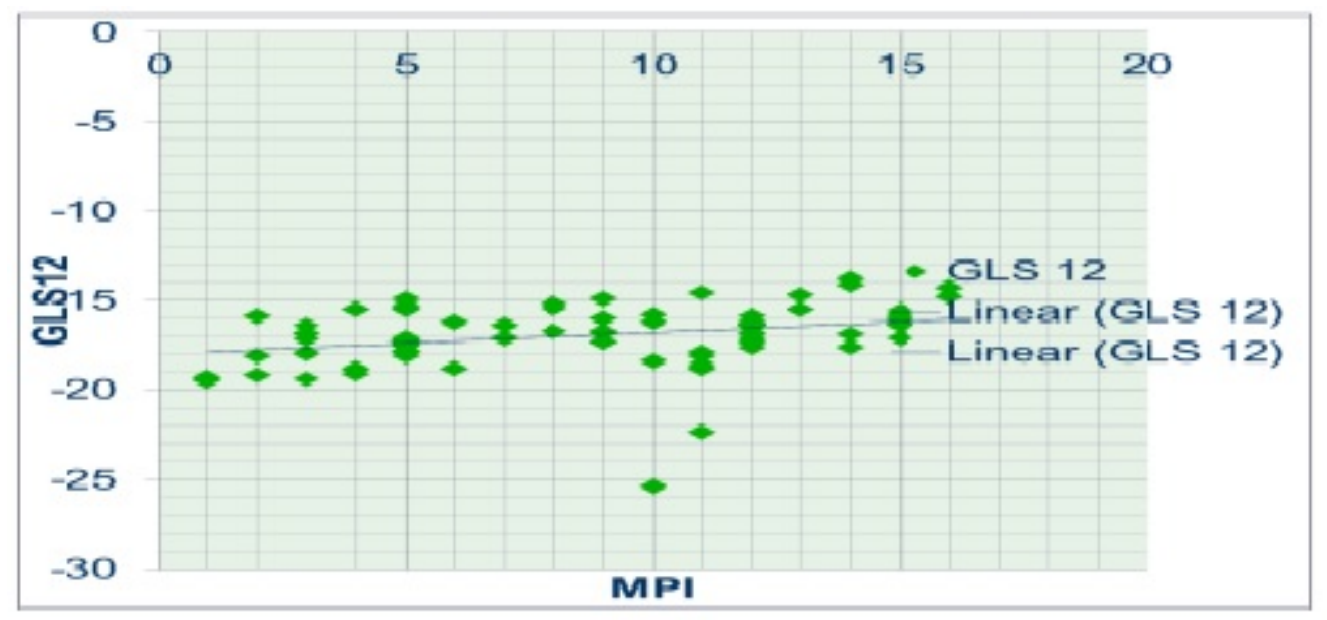

Figure 1: MPI vs GLS12.

There was statistically significant difference between patients with single vessel disease $v s$ two vessel disease and multi vessel disease as regard GLSr12 and GLSr17, but most of SLSr were not significant.

In this study, we found that strain parameters at basal anterior (BA), basal antro-septal (BAS), mid-anterior (MA), mid antro-septal (MIS), mid antro-septal (MAS), Apical inferior (AI) and apical lateral (AL) segments were found to be significant predictor of LAD stenosis, Basal posterior (BP) and mid posterior (MP) segments were found to be significant predictor of LCX stenosis and Basal inferior (BI) segment was found to be predictor of RCA stenosis, also we found that strain rate parameters at $\mathrm{BA}, \mathrm{MA}, \mathrm{MAS}, \mathrm{AI}, \mathrm{AL}$ and apex segments were found to be significant predictor of LAD stenosis, at BL, BP and ML segment were found to be significant predictor of LCX stenosis and at BI and MI were found to be predictor of RCA stenosis (Table 2).

\section{Table 2: MPI vs GLS.}

\begin{tabular}{|l|l|l|}
\hline Segments & $r$ & $p$ \\
\hline BA $(M P I) \times B A(S)$ & 0.421 & $<0.001$ \\
\hline BAS(MPI) $\times$ BAS(S) & 0.284 & 0.003 \\
\hline$M A(M P I) \times M A(S)$ & 0.281 & 0.003 \\
\hline MIS(MPI) $\times$ MIS(S) & 0.383 & $<0.001$ \\
\hline$M A S(M P I) \times M A S(S)$ & 0.528 & $<0.001$ \\
\hline$A A(M P I) \times A A(S)$ & 0.717 & $<0.001$ \\
\hline$A S(M P I) \times A S(S)$ & 0.322 & 0.001 \\
\hline$A I(M P I) \times A I(S)$ & 0.558 & $<0.001$ \\
\hline$A L(M P I) \times A L(S)$ & 0.561 & $<0.001$ \\
\hline
\end{tabular}

\begin{tabular}{|l|l|l|}
\hline Apex(MPI) $\times$ Apex(S) & 0.58 & $<0.001$ \\
\hline $\mathrm{BL}(\mathrm{MPI}) \times \mathrm{BL}(\mathrm{S})$ & 0.717 & $<0.001$ \\
\hline $\mathrm{BP}(\mathrm{MPI}) \times \mathrm{BP}(\mathrm{S})$ & 0.38 & $<0.001$ \\
\hline $\mathrm{ML}(\mathrm{MPI}) \times \mathrm{ML}(\mathrm{S})$ & 0.718 & $<0.001$ \\
\hline $\mathrm{MP}(\mathrm{MPI}) \times \mathrm{MP}(\mathrm{S})$ & 0.322 & 0.001 \\
\hline $\mathrm{BI}(\mathrm{MPI}) \times \mathrm{BI}(\mathrm{S})$ & 0.681 & $<0.001$ \\
\hline $\mathrm{BIS}(\mathrm{MPI}) \times \mathrm{BIS}(\mathrm{S})$ & 0.061 & 0.529 \\
\hline $\mathrm{MI}(\mathrm{MPI}) \times \mathrm{MI}(\mathrm{S})$ & 0.713 & $<0.001$ \\
\hline
\end{tabular}

In this study, we found that there was no correlation between the total number of segments affected in MPI and results of coronary angiography, $\mathrm{P}$ value was not significant for the summation of total segments, however it was significant for LAD territory.

There was a positive correlation between the number of segments affected in MPI and GLS17, GLS12, GLSr17 and GLSr12 with a significant P value for GLS12 and GLSr12 but not for GLS17 or GLSr17. The results are showing a positive correlation between the 17 segments comparing MPI and SLSS and SLSr parameters of speckle tracking study with a significant $\mathrm{P}$ value (Table 3 ).

\section{Table 3: MPI vs GLS.}

\begin{tabular}{|l|l|l|}
\hline Segments & $r$ & \multicolumn{1}{l|}{ p } \\
\hline $\mathrm{BA}(\mathrm{MPI}) \times \mathrm{BA}(\mathrm{Sr})$ & 0.394 & $<0.001$ \\
\hline $\mathrm{BAS}(\mathrm{MPI}) \times \mathrm{BAS}(\mathrm{Sr})$ & 0.066 & 0.482 \\
\hline $\mathrm{MA}(\mathrm{MPI}) \times \mathrm{MA}(\mathrm{Sr})$ & 0.183 & 0.044 \\
\hline $\mathrm{MIS}(\mathrm{MPI}) \times \mathrm{MIS}(\mathrm{Sr})$ & 0.519 & $<0.001$ \\
\hline
\end{tabular}




\begin{tabular}{|l|l|l|}
\hline $\mathrm{MAS}(\mathrm{MPI}) \times \mathrm{MAS}(\mathrm{Sr})$ & 0.238 & 0.042 \\
\hline $\mathrm{A} A(\mathrm{MPI}) \times \mathrm{AA}(\mathrm{Sr})$ & 0.229 & 0.029 \\
\hline $\mathrm{AS}(\mathrm{MPI}) \times \mathrm{AS}(\mathrm{Sr})$ & 0.197 & 0.036 \\
\hline $\mathrm{AI}(\mathrm{MPI}) \times \mathrm{AI}(\mathrm{Sr})$ & 0.567 & 0.041 \\
\hline $\mathrm{AL}(\mathrm{MPI}) \times \mathrm{AL}(\mathrm{Sr})$ & 0.462 & 0.005 \\
\hline $\mathrm{Apex}(\mathrm{MPI}) \times \mathrm{Apex}(\mathrm{Sr})$ & 0.15 & 0.109 \\
\hline $\mathrm{BL}(\mathrm{MPI}) \times \mathrm{BL}(\mathrm{Sr})$ & 0.03 & 0.748 \\
\hline $\mathrm{BP}(\mathrm{MPI}) \times \mathrm{BP}(\mathrm{Sr})$ & 0.614 & 0.009 \\
\hline $\mathrm{ML}(\mathrm{MPI}) \times \mathrm{ML}(\mathrm{Sr})$ & 0.324 & 0.019 \\
\hline $\mathrm{MP}(\mathrm{MPI}) \times \mathrm{MP}(\mathrm{Sr})$ & 0.005 & 0.957 \\
\hline $\mathrm{BI}(\mathrm{MPI}) \times \mathrm{BI}(\mathrm{Sr})$ & 0.491 & 0.026 \\
\hline $\mathrm{BIS}(\mathrm{MPI}) \times \mathrm{BIS}(\mathrm{Sr})$ & 0.146 & 0.121 \\
\hline $\mathrm{MI}(\mathrm{MPI}) \times \mathrm{MI}(\mathrm{Sr})$ & 0.405 & 0.001 \\
\hline $\mathrm{BA}(\mathrm{MPI}) \times \mathrm{BA}(\mathrm{Sr})$ & 0.394 & $<0.001$ \\
\hline $\mathrm{BAS}(\mathrm{MPI}) \times \mathrm{BAS}(\mathrm{Sr})$ & 0.066 & 0.482 \\
\hline $\mathrm{MA}(\mathrm{MPI}) \times \mathrm{MA}(\mathrm{Sr})$ & 0.183 & 0.044 \\
\hline $\mathrm{MIS}(\mathrm{MPI}) \times \mathrm{MIS}(\mathrm{Sr})$ & 0.519 & $<0.001$ \\
\hline $\mathrm{MAS}(\mathrm{MPI}) \times \mathrm{MAS}(\mathrm{Sr})$ & 0.238 & 0.042 \\
\hline $\mathrm{AA}(\mathrm{MPI}) \times \mathrm{AA}(\mathrm{Sr})$ & & 0.029 \\
\hline & & \\
\hline
\end{tabular}

\section{Discussion}

Subclinical impairment of the LV function has been demonstrated by 2DSE in the setting of many disorders including hypertension, diabetes mellitus [10] atrial fibrillation and heart failure with preserved ejection fraction [11]. Smaller previous studies have demonstrated impaired peak longitudinal strain and strain rate in patients with CAD [2]. The myocardial fibers most susceptible to ischemia are the longitudinally orientated fibers which are located subendocardially. Measurements of longitudinal motion and deformation are therefore the most sensitive markers of CAD [1]. The analysis of myocardial deformation has recently emerged as a quantitative means of reliably estimating myocardial contractility. The strain represents the percentage shortening of the myocardial fibers, while the strain rate, which represents the speed of myocardial deformation is derived as a function of strain and time. The main advantage of this method that it is less dependent on the load than the traditional methods. Other studies demonstrated that color TDI are sensitive markers of longitudinal dysfunction caused by CAD and that these TDI velocities can improve the diagnostics of CAD in patients suspected of stable angina pectoris [12]. However, local myocardial

velocities obtained by TDI have the disadvantage of being influenced by heart movement and tethering to adjacent segments, which makes 2DSE more suitable for diagnosing impaired segmental longitudinal mechanics caused by CAD [13]. Our study was conducted to determine the correlation between 2DSE at rest and MPI in diagnosis of CAD instable angina pectoris patients.

In this study, we found no statistically significant difference between the two groups as regard of left ventricular dimensions and $\mathrm{EF}$, and this was in agreement with Nucifora $G$ et al., and Shimoni $S$ et al., and Biering-Sorensen et al. [2,14-16]. However, statistically significant difference between the two groups as regard of $\mathrm{E}$ wave, $\mathrm{A}$ wave, $\mathrm{E} / \mathrm{A}$ ration, DT, $\mathrm{Em}$ and $\mathrm{E} / \mathrm{Em}$ was found. That is in agreement with Nucifora G et al. and Biering-Sorensen et al. $[15,16]$, Coronary artery disease is considered one of the potential causes of LV diastolic dysfunction. Previous studies indeed showed a high prevalence of global and regional LV diastolic dysfunction in patients with CAD and normal LV systolic function [17]. Moreover, a progressive impairment of LV relaxation has been observed in relation to the severity of coronary atherosclerosis and the number of diseased vessels, and a reversal of these abnormalities has been described after percutaneous coronary intervention [18]. Several mechanisms have been proposed to explain this association. In particular, it has been postulated that repetitive episodes of subclinical ischemia may impair LV relaxation, which is an active, energy-dependent process. In addition, the presence of severely reduced coronary flow may induce structural remodelling of the myocardium (i.e. myocardial fibrosis and hypertrophy and glycogen accumulation), leading to LV diastolic dysfunction [19].

In this study, statistically significant difference was found between the two groups as regard of SLSS and GLS 17 and GLS 12 segments, and this was in agreement with Shimoni $S$ et al. Smedsrud et al. Anwar and Biering-Sorensen et al. $[2,14,16,20]$. The latter study included 86 patients investigated whether the duration of LV early systolic lengthening could accurately identify patients with CAD and also in agreement with Montgomery et al. who retrospectively studied 2D STE characteristics in 123 consecutive patients for assessment of Global longitudinal strain which aids in the detection of non-obstructive coronary artery disease in the resting echocardiogram [21].

Our results also showed statistically significant difference between the two groups as regard of SLSr and GLSr 17and GLSr 12 this was in agreement with Nucifora G et al. and Biering-Sorensen et al. [15,16].

Statistically significant difference between patients with single vessel disease $v s$ two vessel disease and multi vessel disease regarding GLS12 were found but no such 
significant correlation was proved for GLS17 and SLSS, this was in agreement with Choi JO et al., and with Biering-Sorensen et al. [16,22]. The former studied 108 patients to evaluate whether global and segmental PSLS measured by the $2 \mathrm{D}$ speckle tracking method with AFI could be useful for detecting severe $\mathrm{CAD}$ and found Global longitudinal strain was lower in the high-risk group than in the other groups.

Statistically significant difference was also present between patients with single vessel disease $v s$ two vessel disease and multi vessel disease as regard of GLSr12 and GLSr17, but most of SLSr was not significant this was in agreement with Biering-Sorensen et al. Segmental analysis of myocardial perfusion studies is based on certain assumptions concerning coronary anatomy. The nearly universal approach is to assign the anterior wall and septum to the LAD, the lateral wall to the LCX, and the inferior wall to the RCA. However, there is no consensus on the exact borders of each vascular territory as evidenced by the significant variation in quantitative polar maps. These differences may be explained by the normal variability of the coronary anatomy. Many nuclear cardiologists and clinicians believe that the supply of the apex can be subdivided into the individual coronary territories, but this assumption is incorrect. The LAD is the primary vessel supplying the apex in the vast majority of patients [23].

Comparing result from MPI with 2DSE, we found no correlation between the total number of segments affected in MPI and results of coronary angiography and $P$ value was not significant for the summation of total segments but significant for LAD territory. Hacker et al. compared MSCT and MPI in 25 patients with known or suspected CAD. These investigators showed that only 8 of 17 significant stenosis on MSCT (47\%) were associated with abnormal perfusion on MPI [24]. Pereztol-Valdes et al. reported that the most specific segments (anterior, anteroseptal, and all apical segments except the infero-apical) correspond to LAD, but no segment can be exclusively attributed to the RCA. Inferoseptal segments can be attributed to LAD or RCA, inferior and inferolateral segments can be attributed to RCA or LCX, and mid-anterolateral segment can be attributed to LAD or LCX [25]. In our study we found a positive correlation between the total number of segments affected in MPI and 2DSE including GLS17, GLS12, GLSr17 and GLSr12 and P value considered significant for GLS12 and GLSr12 but not for GLS17 and GLSr17, there was also a positive correlation between the 17 segments in MPI and SLSS and SLSr parameters and the $\mathrm{P}$ value considered mostly significant. No previous published study compared longitudinal systolic strain by speckle tracking with ischemic segments by SPECT MPI but most studies directed towards detection of viability, as the study conducted by Roes et al. shows that the longitudinal peak systolic strain parameter achieved a higher diagnostic accuracy in predicting non-viable myocardium [26]. Martin et al. who studied 100 patients with chronic ischemic left ventricular (LV) dysfunction for assessment of myocardial viability using STE and rest SPECT and found that STE is more accurate in predicting non-viable myocardium [27]. Ola et al. reported that Global strain measured by $2 \mathrm{D}-\mathrm{STE}$ is an excellent predictor of myocardial infarct size in chronic ischemic heart disease. Territorial strain is a specific index of the infracted coronary artery. Peak systolic strain measured by 2DSTE discriminates between non-infarcted, transmural infarcted and subendocardial-infarcted segments [28].

Correlation between the affected artery and identified segment using strain parameters showed that BA, BAS, MA, MIS, MAS, AI and AL segments were found to be significant predictor of LAD stenosis and $\mathrm{BP}$ and MP are significant predictor of LCX stenosis while BI is a predictor to RCA stenosis, also we found that strain rate parameters at BA, MA, MAS, AI, AL and apical segments were found to be significant predictor of LAD stenosis, BL, BP and ML as predictor of LCX stenosis while $\mathrm{BI}$ and $\mathrm{MI}$ are predictor of RCA stenosis and that was in agreement with Choi JO et al. and Biering-Sorensen et al. [16,22].

\section{Study Limitation}

The accuracy of STE largely depends on image quality. However, many patients were excluded from our study because of inadequate image quality. Radial, circumferential strain was not performed in the present study. However, the myocardial fibers most susceptible to ischemia are the longitudinally orientated fibers that are located subendocardially, that are why measurements of longitudinal deformation are thought to be the most sensitive markers of CAD. Wall motion score index was not determined in the present study as the patients enrolled had no prior history of cardiac disease, prevalence of cardiac risk factors was relatively low, and conventional Echocardiography was normal, determined by a normal LVEF, we do not think that visually impaired wall motion abnormalities would be frequent. The assessment of coronary angiography was done qualitatively by a single cardiologist who was blinded to the results of strain analysis. Territorial strain is based on a schematic distribution of territories and thus ignores the individual variation in coronary topography and dominance.

\section{Recommendations}

Patients with chest pain and inconclusive ECG findings should undergo Echocardiographic examinations with strain analysis as early as possible, both to facilitate early exclusion of significant coronary disease and thus lead to early discharge and overall cost savings, also less exposure to radiation and ionizing 
contrast material of MPI and Coronary angiography. We recommended this study to be extended in a large scale to validate our observations.

\section{Conclusion}

Myocardial strain by speckle tracking is superior to conventional Echo. Parameters measurements of global and segmental LS using 2DSE and it is a more sensitive tool in the identification of WMA at rest than visual analysis and that support its use to risk stratify atherosclerotic CAD. It may help in identifying which coronary artery is affected. It is found-and for the first time-that 2DSE is not inferior to the MPI in the noninvasive diagnosis of CAD.

Background and methods: In this work, 120 patients suspected of having stable angina pectoris were included. They were presented for evaluation of chest pain and to whom clinical evaluation, echocardiography, nuclear scanning and coronary angiography were done. They were classified into group (A) 40 control patients considered as a control group with normal coronaries, and group (B) 80 patients with significant CAD.

Results: The study showed that regarding the Echo. Parameters, there were statistically significant difference between the 2 groups regarding the A wave, E/A ration, DT, Em and E/EM. Also regarding SLSS and GLS 17 and GLS 12 as well as SLSr, GLSr 17 and GLSr 12. Significant difference was present regarding number of vessels affected in regard to GLS 12, GLSr 12 and GLSr 17. In comparison with the results of MPI, there was a positive correlation between the number of segments affected in MPI and GLS 12 and GLSr 12. A statistically significant correlation was also found between the 17 segments in MPI and SLSS and SLSr parameters.

Conclusion: Myocardial strain by speckle tracking is superior to conventional Echo. Parameters measurements of global and segmental LS using 2DSE and it is a more sensitive tool in the identification of WMA at rest than visual analysis and that support its use to risk stratify atherosclerotic CAD. It may help in identifying which coronary artery is affected.

\section{References}

1. Hoffmann S, Jensen JS, Iversen AZ, et al. Tissue Doppler echocardiography improves the diagnosis of coronary artery stenosis instable angina pectoris. Eur. Heart. J. Cardiovasc. Imaging. 13, 724-729 (2012).

2. Shimoni S, Gendelman G, Ayzenberg O, et al. Differential effects of coronary artery stenosis on myocardial function: the valueof myocardial strain analysis for the detection of coronary artery disease. J. Am. Soc. Echocardiogr. 24, 748757 (2011).

3. Ciaroni S, Bloch A, Hoffmann JL, Bettoni M, Fournet D. Prognostic value of dobutamine echocardiography in patients with intermediate coronary lesions at angiography. Echocardiogr. 19, 549-553 (2002).

4. Lang RM, Bierig M, Devereux RB. Recommendations for chamber quantification: a report from the American Society of Echocardiography's Guidelines and Standards Committee and the Chamber Quantification Writing Group, developed in conjunction with the European Association of Echocardiography, a branch of the European Society of Cardiology. J. Am. Soc. Echocardiogr. 18, 1440-1463 (2005).

5. Nagueh SF, Middleton KJ, Kopelen HA, Zoghbi WA, Quinones MA. Doppler tissue imaging: a non-invasive technique for evaluation of left ventricular relaxation and estimation of filling pressures. J. Am. Coll. Cardiol. 30, 1527-1533 (1997).

6. Nagueh SF, Lakkis NM, Middleton KJ, Spencer WH III, Zoghbi WA, Quinones MA. Doppler estimation of left ventricular filling pressures in patients with hypertrophic cardiomyopathy. Circulation. 99, 921-926 (1999).

7. Anwar AM. Global and segmental myocardial deformation by 2D speckle tracking compared to visual assessment. W. J. Cardio. 4(12), 341-346 (2012).

8. Cerqueira MD, Allman KC, Ficaro EP, et al. Recommendations for reducing radiation exposure in myocardial perfusion imaging. J. Nucl. Cardiol. 17, $709-718$ (2010).

9. Eagle KA, Guyton RA, Davidoff R, et al. ACC/AHA 2004 guideline update for coronary artery bypass graft surgery: summary article: a report of the American College of Cardiology/American Heart Association Task Force on
Practice Guidelines (Committee to Update the 1999 Guidelines for Coronary Artery Bypass Graft Surgery). Circulation. 110, 1168-1176 (2004).

10. Chung J, Abraszewski P, Yu X, et al. Paradoxical increase in ventricular torsion and systolic torsion rate in type Idiabetic patients under tight glycemic control. J. Am. Coll. Cardiol. 47, 384-390 (2006).

11. Tan YT, Wenzelburger F, Lee E, et al. The pathophysiology of heart failure with normal ejection fraction: exercise echocardiography reveals complex abnormalities of both systolic and diastolic ventricular function involvingtorsion, untwist, and longitudinal motio. J. Am. Coll. Cardiol. 54, 36-46 (2009).

12. Hoffmann S, Mogelvang R, Sogaard P, et al. Tissue Doppler echocardiography reveals impaired cardiac function in patients with reversible ischaemia. Eur. J. Echocardiogr. 12, 628-634 (2011).

13. Urheim S, Edvardsen T, Torp H, Angelsen B, Smiseth OA. Myocardial strain by Doppler echocardiography. Validation of a new method to quantify regional myocardial function. Circulation. 102, 1158-1164 (2000).

14. Anwar AM. Accuarcy of two-dimensional speckle tracking echocardiography for the detection of significant coronary stenosis. J. Cardiovasc. Ultrasound. 21, 177-182 (2013).

15. Nucifora G, Schuijf JD, Delgado V, et al. Incremental value of subclinical leftventricular systolic dysfunction for the identification of patients with obstructive coronaryartery disease. Am. Heart J. 159, 148-157 (2010).

16. Biering-Sorensen $\mathrm{T}$, Hoffman $\mathrm{S}$, Mogelvang $\mathrm{R}$, et al. Myocardial strain analysis by 2-Dimensional speckle tracking echocardiography improves diagnostics of coronary artery stenosis instable angina pectoris. Circ. Cardiovasc. Imaging. 7, 58-65 (2014).

17. Liang HY, Cauduro S, Pellikka P, et al. Usefulness of twodimensional speckle strain for evaluation of left ventricular diastolic deformation in patients with coronary artery disease. Am. J. Cardiol. 98, 1581-1586 (2006).

18. Tanaka H, Kawai H, Tatsumi K, et al. Improved regional myocardial diastolic function assessed by strain rate imaging in patients with coronary artery disease undergoing percutaneous coronary intervention. J. Am. Soc. Echocardiogr. 19, 756 -762 (2006). 
19. Yuda S, Fang ZY, Marwick TH. Association of severe coronary stenosis with subclinical left ventricular dysfunction in the absence of infarction. J. Am. Soc. Echocardiogr. 16, 1163-1170 (2003).

20. Smedsrud MK, Sarvari S, Haugaa KH, et al. Duration of myocardial early systolic lengthening predicts the presence of significant coronary artery disease. J. Am. Coll. Cardiol. 60, 1086-1093 (2012).

21. Montgomery DE, Puthumana JJ, Fox JM, Ogunyakin KO. Global longitudinal strain aids the detection of nonobstructive coronary artery disease in the resting echocardiogram. Eur. Heart. J. Cardiovasc. Imaging. 13, 579587 (2012)

22. Choi JO, Cho SW, Song YB, et al. Longitudinal 2D strain at rest predicts the presence of left main and three vessel coronary artery disease in patients without regional wall motion abnormality. Eur. J. Echocardiogr. 10, 695-701 (2009).

23. Alderman EL, Stadius M. The angiographic definitions of the bypass angioplasty revascularization investigation. Coronary. Artery. Disease. 3, 1189-1207 (1992).

24. Hacker M, Jakobs T, Matthiesen F. Comparison of spiral multidetector CT angiography and myocardial perfusion imaging in the noninvasive detection of functionally relevant coronary artery lesions: first clinical experiences. J. Nucl. Med. 46, 1294-1300 (2005).

25. Pereztol-Valdes O, Candell-Riera J, Santana-Boado C, et al. Correspondence between left ventricular 17 myocardial segments and coronary arteries. Eur. Heart J. 26, 2637-2643 (2005).

26. Roes SD, Mollema SA, Lamb HJ, vanderWall EE, de Roos A, Bax JJ. Validation of echocardiographic two-dimensional speckle tracking longitudinal strain imaging for viability assessment in patients with chronic ischemic left ventricular dysfunction and comparison with contrast-enhanced magnetic resonance imaging. Am. J. Cardiol. 104(3), 312-317 (2009).

27. Hutyra, Skala, Kaminek, et al. Speckle tracking echocardiography derived systolic longitudinal strain is better than rest single photon emission tomography perfusion imaging for nonviable myocardium identification. Biomed. Pap. Med. 157(1), 12-21 (2013).

28. Ola, Einar, Trond, et al. Global longitudinal strain measured by two dimensional speckle tracking echocardiography is closely related to myocardial infarct size in chronic ischaemic heart disease. Clinical. Science. 113, 287-296 (2007). 